\title{
Evaluation of random forests performance for genome-wide association studies in the presence of interaction effects
} Yoonhee Kim ${ }^{1}$, Robert Wojciechowski ${ }^{1}$, Heejong Sung ${ }^{1}$, Rasika A Mathias ${ }^{1}$, Li Wang ${ }^{1,2}$, Alison P Klein ${ }^{1,2}$, Rhoshel K Lenroot ${ }^{3}$, James Malley ${ }^{4}$ and Joan E Bailey-Wilson*1

\author{
Addresses: ${ }^{1}$ National Human Genome Research Institute, National Institutes of Health, 333 Cassell Drive, Baltimore, MD 21224, USA, \\ ${ }^{2}$ Johns Hopkins University School of Medicine, 733 North Broadway, Baltimore, Maryland 21205, USA, ${ }^{3}$ National Institute of Mental Health, \\ National Institutes of Health, 6001 Executive Boulevard, Bethesda, Maryland 20892-9663, USA and ${ }^{4}$ Center for Information Technology, \\ National Institutes of Health, 10401 Fernwood Road, Bethesda, Maryland 20817, USA \\ E-mail: Yoonhee Kim - kimyoo@mail.nih.gov; Robert Wojciechowski - robwoj@mail.nih.gov; Heejong Sung - sunghe@mail.nih.gov; \\ Rasika A Mathias - rmathias1@mail.nih.gov; Li Wang - liwang@jhsph.edu; Alison P Klein - aklein1@jhmi.edu; \\ Rhoshel K Lenroot - lenrootr@mail.nih.gov; James Malley - jmalley@mail.nih.gov; Joan E Bailey-Wilson* - jebw@mail.nih.gov \\ ${ }^{*}$ Corresponding author
}

from Genetic Analysis Workshop 16

St Louis, MO, USA 17-20 September 2009

Published: 15 December 2009

BMC Proceedings 2009, 3(Suppl 7):S64 doi: 10.1186/1753-656I-3-S7-S64

This article is available from: http://www.biomedcentral.com/I753-656I/3/S7/S64

(C) 2009 Kim et al; licensee BioMed Central Ltd.

This is an open access article distributed under the terms of the Creative Commons Attribution License (http://creativecommons.org/licenses/by/2.0), which permits unrestricted use, distribution, and reproduction in any medium, provided the original work is properly cited.

\begin{abstract}
Random forests (RF) is one of a broad class of machine learning methods that are able to deal with large-scale data without model specification, which makes it an attractive method for genome-wide association studies (GWAS). The performance of RF and other association methods in the presence of interactions was evaluated using the simulated data from Genetic Analysis Workshop 16 Problem 3, with knowledge of the major causative markers, risk factors, and their interactions in the simulated traits. There was good power to detect the environmental risk factors using RF, trend tests, or regression analyses but the power to detect the effects of the causal markers was poor for all methods. The causal marker that had an interactive effect with smoking did show moderate evidence of association in the RF and regression analyses, suggesting that RF may perform well at detecting such interactions in larger, more highly powered datasets.
\end{abstract}

\section{Background}

Random forests (RF) [1] is one of a broad class of machine learning methods that are able to deal with large-scale data without precise model specification: it is massively nonparametric. It performs random searches through feature space and data space, the latter by using bootstrap sampling. It generates multiple recursively partitioned classification trees (the exact number determined by the analyst), called a "forest". RF has gained attention as a method that may be useful for detecting associations when there are large numbers of predictor variables to be evaluated, such as single-nucleotide 
polymorphism (SNP) loci in a genome-wide association study (GWAS). It has also been suggested that RF may perform better than other methods when the causative loci have minimal marginal effects but larger interaction effects [2-6]. The calculation of importance values and the very local nature of the classification in each tree allows RF to automatically evaluate gene-gene and geneenvironmental interactions [5] without assuming complex models or explicitly testing all possible interactions. Many GWAS test each locus under the generally implausible assumption of complete or weak statistical independence across all loci. RF evaluates the predictive strength of all loci by averaging the importance of each in all possible SNP-SNP and SNP-covariate contexts.

In many genetic association studies, a common approach is to follow up the most significant results from a GWAS by genotyping independent samples for a smaller number of SNPs (e.g., 1536 or 3072) using customized arrays [7]. At this point, it is unknown whether RF would be more likely than standard regression methods to include true causative SNPs in the second stage of genotyping in the presence of genegene or gene-environment interactions in GWAS data.

To address this issue, the Genetic Analysis Workshop 16 (GAW16) Problem 3 simulated data set was used to evaluate performance of RF and several other association methods. Given knowledge of the major causative SNPs and risk factors for these simulated traits [8], we compared whether the major risk factors were detected in the RF analyses performed in the statistical package $\mathrm{R}$ to standard association tests in the computer program PLINK [9], and backward-elimination RF [3], which iteratively eliminates a pre-specified portion of predictors based on low importance values until the error rates of test datasets (out-of-bag samples) minimize to a certain point. Methods were evaluated based on whether the associated SNPs were among the most significant set of SNPs to be selected for genotyping at a hypothetical "second stage".

\section{Methods}

The Framingham Heart Study SHARe dataset for GAW16 Problem 2 formed the basis of the simulated data pedigree structures: 6,476 individuals with both phenotype and genotype data in 942 pedigrees across three generations plus 188 unrelated individuals. The genotypes for all Problem three replicates were fixed to those that were actually observed for the Framingham Heart Study SHARe participants. Phenotypes were simulated for all genotyped individuals at three time points, 10 years apart. Total cholesterol (CHOL) was defined as the sum of the simulated phenotypes triglyceride, high-density lipoprotein (HDL), and low-density lipoprotein (LDL). The traits were simulated such that over time, one develops cranial adipose cumulation (CAC). People with high levels of CAC were at higher risk for myocardial infarction (MI). Smoking also increased the risk of MI in these simulated data. The MI trait was simulated so that the age-adjusted incidence of MI was higher for men than for women. The MI and CAC traits, along with the strongest causative risk factors on the etiologic pathway used in the simulation, are detailed in Kraja et al. [8]. Two hundred replicate datasets were created based on the same generating model.

\section{Genotype quality control}

In the $50 \mathrm{k}$-SNP panel (48,071 SNPs), quality control measures were performed in the following order: 1) individuals with genotype calls for $<95 \%$ of the SNPs were removed; 2) all genotypes with a confidence score (the probability that a given genotype is accurate, provided by the BRLLM allele-calling algorithm $[10,11])<0.95$ were considered as missing; 3) SNPs with call rates $<98 \%$ or minor allele frequencies (MAF) $<1 \%$ were removed. 4) An additional 1,317 SNPs departing from Hardy-Weinberg proportions $(p<0.01)$ were excluded, leaving 31,538 SNPs for the analyses. Markers showing mendelian inconsistencies were coded as unknown in the parent(s).

\section{Phenotype definition}

Phenotype observations from the first two replicates in the simulated data were combined for case and control selection (three phenotype time-points per replicate) using both the family data and the unrelated individuals (singletons) as described below. Because the number of unrelated cases with an MI in any one replicate was small, these two replicates were combined to increase the power to detect variables that contribute to risk of the simulated disease by increasing the number of cases. Subjects were classified as a "case" if they had at least one MI and as a "control" if they were free from any MI over all six time-points. Families were classified as "casefamilies" if they contained at least one case and "controlfamilies" if they had no cases. Among the genotyped persons, 563 unrelated cases (329 males) and 553 unrelated controls (243 males) were selected. These included all singletons in addition to the youngest case from each case family and the oldest control from each control family. Environmental risk factors (age, sex, smoking, and $\mathrm{CHOL}$ ) and $\mathrm{CAC}$ were taken from the visit that corresponded to the earliest MI for cases and the final visit in the first replicate for controls. Where a case had an MI in both replicates at the same time point, phenotypes from the first replicate were selected. These 1,116 persons were used in all analyses described here. 


\section{RF analyses}

Imputation of missing genotypes (1\% of genotypes) was performed using the RfImpute function implemented in randomForest package 4.5 in $\mathrm{R}$ 2.7.1. RF analyses were run 100 times, randomly sampling 31,000 SNPs in each run with 1000 trees per forest. Each RF model included covariates of age in years, sex, CHOL, and smoking status (yes, no) as potential predictors. Analyses were performed for MI as a dichotomous trait for RF classification and CAC as a quantitative trait for RF regression. Each of these analyses was performed in two ways, using either 200 or 7 randomly sampled predictors at each node (mtry option in the randomForest package) in each tree.

The results over the $100 \mathrm{RFs}$ were summarized by counting the number of times the major causative loci for the CAC and MI traits appeared in the subsets of SNPs identified by each RF as being most predictive based on importance indices generated by randomForest [1]: GINI (the sum over all trees of the decrease in Gini impurity after each split) and mean decrease in accuracy (MDA) for classification or mean decrease in mean squared error (MSE) for regression. These subsets included either 1536 or 3072 SNPs to match the numbers of SNPs often used for second stage genotyping in GWAS studies. Backward-elimination variable selection was performed using the varSelRF 0.6-5 package with the corresponding RF options as described above (1000 trees per forest) but discarding 40\% of the lowest ranked predictors at the start of each new forest.

\section{PLINK analyses}

Case-control (Cochrane-Armitage trend) and quantitative-trait (linear regression) association analyses were performed in PLINK [9]. Multivariate linear and logistic regression, adjusting for linear effects of age, sex, smoking status, and CHOL, were also used under additive genetic and log-additive models. For regression analyses, $p$-values were obtained using Wald $t$-tests; for the Cochrane-Armitage test, they were derived from the asymptotic chi-squared distribution (1 df).

\section{Results and discussion}

For comparison with our RF analyses, standard significance tests for association were performed for MI and $\mathrm{CAC}$, adjusting for covariates using PLINK. Among the SNPs that were simulated to have the strongest genetic effects on MI and CAC, none showed genome-wide significance, and only rs12565497 $(p=0.003)$ showed a nominal significance level (MI below 0.01) (Tables 1 and 2). In multivariate linear regression, both sex and CHOL were significantly correlated with CAC. In multivariate logistic regression, age, sex, smoking status, and CHOL were highly associated with MI. The lowest $p$-value among causal markers was $p=0.000192$ for rs12565497. This SNP ranked $16^{\text {th }}$ among all 31,538 markers tested with multivariate logistic regression. Two other causal SNPs (rs17714718 and rs1894638) would have been retained for second-stage analysis using the univariate rankings (at 1549 and 2683, respectively), but not based on the multivariate analysis.

In the main RF analyses, we compared several analysis strategies. First, we compared which importance score was best to use for ranking SNPs (MDA vs. GINI (MSE)). We observed that the causal SNPs and important covariates were more likely to be included in the top predictor lists to pass to second-stage analysis when the ranking was based on the GINI index for classification (REP in Table 1) or MSE index for regression (REP in Table 2) than when based on MDA scores. For example, rs12565497 appeared in the TOP 3072 list in $71 \%$ of the 100 forests when using GINI compared with $13 \%$ using the MDA index (data not shown). It should be noted that Strobl et al. [12] have shown that there is bias in several of the variable-selection processes (including GINI and a permutation accuracy importance measure) often used in RF analyses when there are quantitative traits on different scales or categorical predictors with different numbers of categories, such that non-causative continuous variables and non-causative variables with large numbers of categories are more likely to be selected as important predictor variables than are variables with small numbers of categories. Because no variables with large numbers of categories were used in this study and the only two continuous variables included in the analysis were known to have an effect on the simulated trait, this sort of bias would not be observed here. However, this potential bias should be accounted for in a study of real data, as suggested by Strobl et al. [12]. Results presented here used categorical (genotypic) coding of genotypes, but additive coding gave similar results.

Table 1 shows that for MI, all environmental risk factors were included in the lists of top predictors in $100 \%$ of the 100 forests when using the mtry option $=200$. For CAC, only age and CHOL ranked highly in all forests. This is reasonable because the simulation framework of MI incorporated smoking status, CAC (and therefore CHOL), and age as risk factors, whereas CAC itself had only age and CHOL as direct risk factors. Age and sex were significantly different in cases and controls $(p=$ $2.2 \times 10^{-16}$ and $1.72 \times 10^{-6}$, respectively). RF performed very well in detecting these environmental risk factors that had comparatively large effects on the traits. RF analyses that included these risk factors had lower mean prediction error rates in $100 \mathrm{RFs}$ ( mean $=23 \%$ and $40 \%$ for mtry $=200$ and 7 , respectively) than did RF analyses that did not include these covariates (mean $=35 \%$ and $43 \%$ for mtry $=200$ and 7 , respectively). 
Table I: PLINK and RF results for MI

\begin{tabular}{|c|c|c|c|c|c|c|}
\hline \multirow[b]{3}{*}{ Predictors } & \multirow{2}{*}{\multicolumn{2}{|c|}{$\begin{array}{c}\text { PLINK }^{a} \\
\begin{array}{c}\text { p-Value } \\
\text { (rank) }\end{array}\end{array}$}} & \multicolumn{4}{|c|}{$\mathbf{R F}$} \\
\hline & & & \multicolumn{2}{|c|}{$\begin{array}{c}\text { REPc } \\
\text { Top I536 }\end{array}$} & \multicolumn{2}{|c|}{$\begin{array}{c}\text { REPc } \\
\text { Top } 3072\end{array}$} \\
\hline & $\begin{array}{c}\text { Univariate } \\
\text { analysis }\end{array}$ & $\begin{array}{c}\text { Multivariate } \\
\text { analysis }\end{array}$ & mtry $=200^{d}$ & mtry $=7^{d}$ & mtry $=200^{d}$ & mtry $=7^{d}$ \\
\hline \multicolumn{7}{|l|}{ Covariates } \\
\hline Age & - & $7.66 \times 10^{-7}$ & 100 & 100 & 100 & 100 \\
\hline Sex & - & $6.97 \times 10^{-12}$ & 100 & 36 & 100 & 44 \\
\hline Smoking & - & $1.72 \times 10^{-10}$ & 100 & 71 & 100 & 84 \\
\hline Cholesterol & - & $<1.0 \times 10^{-50}$ & 100 & 100 & 100 & 100 \\
\hline \multicolumn{7}{|l|}{ CAC } \\
\hline $\begin{array}{c}r s 6743961 \\
(M A F=0.49)\end{array}$ & $\begin{array}{c}0.45 I \\
(16568)\end{array}$ & $\begin{array}{c}0.7394 \\
(24158)\end{array}$ & 2 & 10 & 9 & 22 \\
\hline $\begin{array}{c}r s|77| 47 \mid 8 \\
(M A F=0.49)\end{array}$ & $\begin{array}{l}0.0188 \\
(1549)\end{array}$ & $\begin{array}{c}0.093 \\
(3893)\end{array}$ & 20 & 13 & 33 & 29 \\
\hline $\begin{array}{c}r s \mid 894638 \\
(M A F=0.49)\end{array}$ & $\begin{array}{l}0.0406 \\
(2683)\end{array}$ & $\begin{array}{l}0.3198 \\
(11586)\end{array}$ & 5 & 18 & 14 & 32 \\
\hline $\begin{array}{c}r s 1919811 \\
(M A F=0.49)\end{array}$ & $\begin{array}{l}0.2106 \\
(9215)\end{array}$ & $\begin{array}{l}0.2088 \\
(8004)\end{array}$ & 8 & 14 & 9 & 21 \\
\hline $\begin{array}{c}r s 213952 \\
(M A F=0.20)\end{array}$ & $\begin{array}{l}0.3923 \\
(14807)\end{array}$ & $\begin{array}{l}0.2319 \\
(8758)\end{array}$ & 11 & 3 & 25 & 11 \\
\hline \multicolumn{7}{|l|}{ MI } \\
\hline $\begin{array}{c}r s \mid 2565497 \\
(M A F=0.30)\end{array}$ & $\begin{array}{c}0.00303 \\
(370)\end{array}$ & $\begin{array}{c}0.000192 \\
(16)\end{array}$ & 56 & 21 & 71 & 37 \\
\hline $\begin{array}{c}r s|l 92755| \\
(M A F=0.29)\end{array}$ & $\begin{array}{c}0.963 \\
(30577)\end{array}$ & $\begin{array}{l}0.6993 \\
(22956)\end{array}$ & I & 4 & 7 & 9 \\
\hline
\end{tabular}

${ }^{\text {a }}$ LINK analyses assumed either additive or log-additive genetic models.

${ }^{b} p$-Values for covariates were averages across all SNPs.

CREP: the number of times in 100 RFs (1000 trees each) in which the given SNP of interest appeared in the top I536 or 3072 predictors based on GINI index.

${ }^{d}$ mtry: the number of predictors (200 or 7 ) randomly selected at each node to find the best split while growing trees.

As expected, the number of times that causal SNPs were ranked high enough to be selected for second-stage analysis (top lists) across the 100 RFs increased with the number of SNPs included in the top lists. Only rs12565497, which was simulated to interact with smoking to affect risk of MI [8], had a moderately high frequency $(71 \%)$ of being in the top 3072 predictors across the 100 RFs. No other SNPs were included in the top 3072 predictors in more than $33 \%$ of the $100 \mathrm{RFs}$ (Table 1). None of the five causal SNPs were included frequently in the top 3072 predictors for CAC, indicating very low statistical power to detect association. Using a larger number $(\mathrm{mtry}=200)$ of predictors at each node rather than a small number $(m+r y=7)$ resulted in better performance as measured by the number of times that causal SNPs were included in the top 3072 SNPs across the 100 RFs. Examination of the top 100 predictors over the $100 \mathrm{RFs}$ for each trait revealed that none of these
SNPs were closer than $4 \mathrm{Mbp}$ to any of the causative loci and none were in linkage disequilibrium with any of the causative loci, thus showing that proxy SNPs for the causal loci were also not serving as strong predictors in the RF analyses. RF using the backward-elimination algorithm took 1 day per $1 \mathrm{RF}$, and the lists of top predictors for MI included all environmental risk factors, but none of the causal SNPs (data not shown).

\section{Conclusions}

These RF results supported the use of the GINI (MSE) index rather than MDA, a large number of sampled predictors per node (i.e., mtry option number in randomForest), and categorical coding of genotype data when using RF for the first stage of a GWAS. In this fairly small sample, the strategy of picking random samples of the available SNPs for each RF analysis and 
Table 2: PLINK and RF results for CAC

\begin{tabular}{|c|c|c|c|c|c|c|}
\hline \multirow[b]{3}{*}{ Predictors } & \multirow{2}{*}{\multicolumn{2}{|c|}{$\begin{array}{c}\text { PLINK }^{a} \\
\begin{array}{c}\text { p-Value } \\
\text { (rank) }\end{array}\end{array}$}} & \multicolumn{4}{|c|}{ RF } \\
\hline & & & \multicolumn{2}{|c|}{$\begin{array}{c}\text { REPc } \\
\text { Top I536 }\end{array}$} & \multicolumn{2}{|c|}{$\begin{array}{c}\text { REPc } \\
\text { Top } 3072\end{array}$} \\
\hline & $\begin{array}{c}\text { Univariate } \\
\text { analysis }\end{array}$ & $\begin{array}{c}\text { Multivariate } \\
\text { analysis }\end{array}$ & mtry $=200^{d}$ & mtry $=7^{d}$ & mtry $=200^{d}$ & mtry $=7^{d}$ \\
\hline \multicolumn{7}{|l|}{ Covariates } \\
\hline Age & - & 0.508 & 100 & 65 & 100 & 65 \\
\hline Sex & - & $1.79 \times 10^{-13}$ & 13 & 6 & 19 & 6 \\
\hline Smoking & - & 0.259 & 11 & 7 & 17 & 7 \\
\hline Cholesterol & - & $<1.0 \times 10^{-50}$ & 100 & 100 & 100 & 100 \\
\hline \multicolumn{7}{|l|}{ CAC } \\
\hline $\begin{array}{c}r s 6743961 \\
(M A F=0.49)\end{array}$ & $\begin{array}{l}0.113 \\
(5427)\end{array}$ & $\begin{array}{c}0.588 \\
(19314)\end{array}$ & 6 & 7 & 11 & 11 \\
\hline $\begin{array}{c}r s \mid 7714718 \\
(M A F=0.49)\end{array}$ & $\begin{array}{l}0.0299 \\
(1940)\end{array}$ & $\begin{array}{l}0.1392 \\
(5287)\end{array}$ & 3 & 5 & 7 & 8 \\
\hline $\begin{array}{c}r s \mid 894638 \\
(M A F=0.49)\end{array}$ & $\begin{array}{l}0.0129 \\
(1024)\end{array}$ & $\begin{array}{c}0.5723 \\
(18983)\end{array}$ & 4 & 9 & 5 & 12 \\
\hline $\begin{array}{c}r s 1919811 \\
(M A F=0.49)\end{array}$ & $\begin{array}{l}0.128 \\
(5948)\end{array}$ & $\begin{array}{l}0.1413 \\
(535 I)\end{array}$ & 5 & 5 & 7 & 6 \\
\hline $\begin{array}{c}r s 213952 \\
(M A F=0.20)\end{array}$ & $\begin{array}{l}0.0786 \\
(4094)\end{array}$ & $\begin{array}{l}0.4738 \\
(15929)\end{array}$ & 4 & 6 & 8 & 14 \\
\hline
\end{tabular}

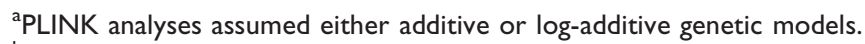

${ }^{b} p$-Values for covariates were averages across all SNPs.

CREP: the number of times in 100 RFs (1000 trees each) in which the given SNP of interest appeared in the top I536 or 3072 predictors based on MSE index.

${ }^{d}$ mtry: the number of predictors (200 or 7$)$ randomly selected at each node to find the best split while growing trees.

then averaging results over 100 forests was not very successful in including the major causative SNPs in the top-ranked sets of SNPs so that they would be included in later replication analyses - only one causal SNP occurred in the top list of SNPs frequently. However, the performance of the RF and regression analyses were very similar and the results suggest that RF may perform well at detecting interactions when the sample size is larger and overall power to detect genetic effects is thus larger. Future simulation studies in much larger samples will be required to resolve this question.

\section{List of abbreviations used}

CAC: Cranial adipose cumulation; CHOL: Cholesterol; GAW: Genetic Analysis Workshop; GINI: Gini index; GWAS: Genome-wide association study; HDL: Highdensity lipoprotein; LDL: Low-density lipoprotein; MAF: Minor allele frequency; MDA: Mean decrease in accuracy; MI: Myocardial infarction; MSE: Mean squared error; RF: Random forests; SNP: Single-nucleotide polymorphism.

\section{Competing interests}

The authors declare that they have no competing interests.

\section{Authors' contributions}

YK, RW, RAM, HS helped to design the study, perform analyses and draft the manuscript, LW helped design the study and performed analyses, AK, JM, RKL and JEB-W helped design the study and participated in writing the manuscript.

\section{Acknowledgements}

The Genetic Analysis Workshops are supported by NIH grant ROI GM03 I575 from the National Institute of General Medical Sciences. This research was supported in part by the Intramural Research Program of the National Institutes of Health (NHGRI, NIMH, CIT). Thanks to Alexa JM Sorant, NHGRI, for computer assistance.

This article has been published as part of BMC Proceedings Volume 3 Supplement 7, 2009: Genetic Analysis Workshop 16. The full contents of the supplement are available online at http://www.biomedcentral.com/ $1753-656 \mid / 3$ ? issue=S7.

\section{References}

I. Breiman L: Random forests. Machine Learning 200I, 45:5-32.

2. Bureau A, Dupuis J, Falls K, Lunetta K, Hayward B, Keith PT and Eerdewegh VP: Identifying SNPs predictive of phenotype using random forests. Genet Epidemiol 2005, 28: I7I-182.

3. Diaz-Uriarte $R$ and Alvarez de Andres $S$. Gene selection and classification of microarray data using random forest. $B M C$ Bioinformatics 2006, 7:3-16. 
4. Heidema AG, Boer MAJ, Nagelkerke N, Mariman CME, Van der ALD and Feskens JME: The challenge for genetic epidemiologists: how to analyze large numbers of SNPs in relation to complex diseases. BMC Genet 2006, 7:23-38.

5. McKinney $A B$, Reif MD, Ritchie $D M$ and Moore $H$ : Machine learning for detecting gene-gene interactions. Appl Bioinformatics 2006, 5:77-88.

6. Lunetta K, Hayward L, Segal J and van Eerdewegh P: Screening large scale association study data: exploiting interactions using random forests. BMC Genet 2004, 5:32-45.

7. Perkel J: SNP genotyping: six technologies that keyed a revolution. Nat Methods 2008, 5:447-454.

8. Kraja AT, Culverhouse R, Daw EW, Wu J, Van Brunt A, Province MA and Borecki IB: The Genetic Analysis Workshop I 6 Problem 3: simulation of heritable longitudinal cardiovascular phenotypes based on actual genome-wide singlenucleotide polymorphisms in the Framingham Heart Study. BMC Proc 2009, 3(suppl 7):S4.

9. Purcell S, Neale B, Todd-Brown K, Thomas L, Ferreira ARM, Bender D, Maller J, Sklar P, de Bakker IW, Daly JM and Sham CP: PLINK: A tool set for whole-genome association and population-based linkage analyses. Am J Hum Genet 2007, 81:559-575.

10. Affymetrix Power Tools (APT) Release apt-1.8.6. http://www affymetrix.com/support/developer/powertools/changelog/index. html.

II. Rabbee $\mathrm{N}$ and Speed TP: A genotype calling algorithm for affymetrix SNP arrays. Bioinformatics 2006, 22:7-I2.

12. Strobl C, Boulesteix AL, Zeileis $A$ and Hothorn $T$ : Bias in random forest variable importance measures: illustrations, sources and a solution. BMC Bioinformatics 2007, 8:25-46.

\section{Publish with Bio Med Central and every scientist can read your work free of charge}

"BioMed Central will be the most significant development for disseminating the results of biomedical research in our lifetime. "

Sir Paul Nurse, Cancer Research UK

Your research papers will be:

- available free of charge to the entire biomedical community

- peer reviewed and published immediately upon acceptance

- cited in PubMed and archived on PubMed Central

- yours - you keep the copyright
BioMedcentral 